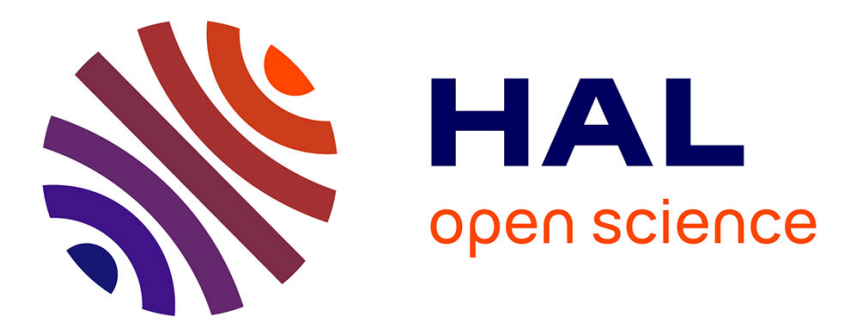

\title{
Numerical modelling of the hydro-chemo-mechanical behaviour of geomaterials in the context of $\mathrm{CO} 2$ injection
}

\author{
V. Vallin, Jean-Michel Pereira, Antonin Fabbri, H. Wong
}

\section{To cite this version:}

V. Vallin, Jean-Michel Pereira, Antonin Fabbri, H. Wong. Numerical modelling of the hydro-chemomechanical behaviour of geomaterials in the context of $\mathrm{CO} 2$ injection. International Journal for Numerical and Analytical Methods in Geomechanics, 2013, 37 (17), pp.3052-3069. 10.1002/nag.2192 . hal-00946115

\section{HAL Id: hal-00946115 \\ https://hal-enpc.archives-ouvertes.fr/hal-00946115}

Submitted on 3 May 2019

HAL is a multi-disciplinary open access archive for the deposit and dissemination of scientific research documents, whether they are published or not. The documents may come from teaching and research institutions in France or abroad, or from public or private research centers.
L'archive ouverte pluridisciplinaire HAL, est destinée au dépôt et à la diffusion de documents scientifiques de niveau recherche, publiés ou non, émanant des établissements d'enseignement et de recherche français ou étrangers, des laboratoires publics ou privés. 


\title{
Numerical modelling of the hydro-chemo-mechanical behaviour of
}

\section{geomaterials in the context of $\mathrm{CO}_{2}$ injection}

\author{
V. Vallin ${ }^{1}$, J.M. Pereira ${ }^{1}$, A. Fabbri ${ }^{2,3}$, H. Wong $^{3}$ \\ ${ }^{1}$ Université Paris-Est, Laboratoire Navier (UMR CNRS-IFSTTAR-ENPC), Ecole des Ponts ParisTech, 6- \\ 8,av.Bl.Pascal,F-77455 Marne-la-Vallée,France, valerie.vallin@enpc.fr, jeanmichel.pereira@enpc.fr \\ ${ }^{2}$ BRGM, Orléans, France (presently at ENTPE) \\ ${ }^{3}$ Université de Lyon, ENTPE/LGCB et LTDS UMR CNRS 5513, Vaulx-en-Velin, France, \\ henry.wong@entpe.fr, antonin.fabbri@entpe.fr
}

KEY WORDS: $\mathrm{CO}_{2}$ geological storage, cement paste, carbonation, hydro-chemo-mechanics, finite volume method

\begin{abstract}
:
Safety assessment of geosequestration of $\mathrm{CO}_{2}$ into deep saline aquifers requires a precise understanding of the study of hydro-chemo-mechanical couplings occurring in the rocks and the cement well. To this aim, a coupled chemo-poromechanical model has been developed and implemented into a research code well-suited to the resolution of fully coupled problems. This code is based on the finite volume methods. In a 1D axisymmetrical configuration, this study aims to simulate the chemo-poromechanical behaviour of a system composed by the cement well and the caprock during $\mathrm{CO}_{2}$ injection. Major chemical reactions of carbonation occurring into cement paste and rocks are considered in order to evaluate the consequences of the presence of $\mathrm{CO}_{2}$ on the amount of dissolved matrix and precipitated calcium carbonates. The dissolution of the solid matrix is taken into account through the use of a chemical porosity. Matrix leaching and carbonation lead, as expected, to important variations of porosity, permeability and to alterations of transport properties and mechanical stiffness. These results justify the importance of considering a coupled analysis accounting for the main chemical reactions. It is worth
\end{abstract}


noting that the modelling framework proposed in the present study could be extended to model the chemo-poromechanical behaviour of the reservoir rock and the caprock when subjected to the presence of an acidic pore fluid $\left(\mathrm{CO}_{2}\right.$-rich brine).

\section{INTRODUCTION}

To meet the challenge of global warming and to the increase of anthropogenic emissions of greenhouse gases into the atmosphere, innovative technologies have to be developed. Among others, geosequestration of carbon dioxide (denoted $\mathrm{CO}_{2}$ hereafter) is considered as one of the most adequate shortterm strategies for achieving important reductions of $\mathrm{CO}_{2}$ emissions into the atmosphere. This technology is based on the capture, transport and sequestration of the carbon dioxide in deep geological formations, such as deep saline aquifers, depleted oil and gas reservoirs or unminable coal seams. The efficiency of the storage relies on the geological configuration which has to include a caprock characterised by a very low permeability and porosity covering a high porosity reservoir rock with sufficient capacity and injectivity. The integrity of the storage could be questioned considering the presence of natural or manmade discontinuities located within the storage complex. In this work, such discontinuities are accounted for by considering the presence of cemented injection or abandoned wells. The presence of $\mathrm{CO}_{2}$ within the injection site will affect the chemical composition of the geomaterials (cement paste and rocks). These chemical reactions will alter the poromechanical properties of the materials, and hence their capacity of confinement. Both in situ studies [1] and laboratory experiments (see for instance [2], [3] and [4]) show the effects of the $\mathrm{CO}_{2}$-rich fluid environments on the chemical compositions and on the poromechanical properties of cement paste and rock samples.

The aim of this study is to simulate the chemo-poromechanical behaviour of wellbore cements in the presence of $\mathrm{CO}_{2}$-rich fluid in the context of $\mathrm{CO}_{2}$ geosequestration in saline aquifers. The cement matrix of the well is composed of several hydrated minerals such as calcium hydrates (mainly Portlandite and hydrated calcium silicates). In the presence of $\mathrm{CO}_{2}$, dissolution and precipitation reactions will occur and largely affect its stiffness and strength. This chemically induced degradation of the cement well is a 
serious threat to the durability of $\mathrm{CO}_{2}$ geosequestration. In consequence, it is important to assess the evolution of chemical reactions which induce alterations on the transport and mechanical properties of the cement paste, in order to estimate the damage risk of the cement well. This work investigates the behaviour of a typical cement well-bore exposed to a $\mathrm{CO}_{2}$-rich in-pore fluid during the post-injection stage. The boundary value problem is solved by assuming 1D axisymmetry under plane strain conditions in the axial direction and infinitesimal transformations. In the first part, the fully-coupled chemoporomechanical model is presented. Its implementation into a finite volume code is then described. Finally, results of the numerical simulations are discussed.

\section{CHEMO-POROMECHANICAL MODEL AND CEMENT PASTE CARBONATION}

In all the study, isothermal conditions are assumed. We consider a two-phase porous medium, composed of a solid phase and a fluid phase. Each of these phases is composed of several species. The solid phase, denoted by S, contains not only the solid cement matrix itself composed of several minerals $\mathrm{M}_{\mathrm{i}}$ but also carbonate crystals $\mathrm{C}$. The in-pore fluid phase $\mathrm{F}$ is made of water, the solvent, denoted by $w$, and dissolved species denoted by $\alpha$ which partly come from the dissolution of the solid phase and partly from dissolved $\mathrm{CO}_{2}$. In the presence of $\mathrm{CO}_{2}$, the main chemical alterations of the cement paste are due to a carbonation process, leading to the leaching of the solid matrix and to the formation of carbonate crystals $\left(\mathrm{CaCO}_{3}\right)$. [5] have shown during experimental carbonation of water saturated Portland-cement paste cores with supercritical $\mathrm{CO}_{2}$ at $90^{\circ} \mathrm{C}$ and $30 \mathrm{MPa}$ the presence of stable and unstable calcium carbonates polymorphs. They observed that vaterite, the least stable $\mathrm{CaCO}_{3}$ polymorph, is located only at the very thin carbonation front, ahead of the carbonated zone, and is transformed into calcite within the main carbonated zone of the cement paste. Furthermore, this transient effect has been neglected and it has been assumed that under the assumption of local equilibrium in the reactive process considered in this study, the calcium carbonates precipitate exclusively as calcite crystals, given the relative stability of this polymorph as compared to other forms (vaterite and aragonite). 


\subsection{Carbonation reactions considered}

The carbonation process considered here is, in agreement with [2], [6], [7], [8] and [9], that of the carbonation of two main hydrates of the cement paste, that is to say Portlandite $\left(\mathrm{Ca}(\mathrm{OH})_{2}\right)$, noted $\mathrm{CH}$, and calcium silicate hydrates, noted $\mathrm{C}-\mathrm{S}-\mathrm{H}$. In standard cement chemistry the notation $\mathrm{C}$ stands for $\mathrm{CaO}, \mathrm{S}$ for $\mathrm{SiO}_{2}$ and $\mathrm{H}$ for $\mathrm{H}_{2} \mathrm{O}$. The "-" sign in $\mathrm{C}-\mathrm{S}-\mathrm{H}$ indicates that no specific composition is implied. In fact, there is a large variety of calcium silicate hydrates depending on the $\mathrm{Ca} / \mathrm{Si}$ ratio, leading to different densities for the $\mathrm{C}-\mathrm{S}-\mathrm{H}$. Hereafter, subscripts following $\mathrm{C}-\mathrm{S}-\mathrm{H}$ characterize the $\mathrm{Ca} / \mathrm{Si}$ ratio.

The first reaction of carbonation corresponds to that of the $\mathrm{CH}$, noted reaction $\left(\mathrm{R}_{1}\right)$, whereas the second one $\left(\mathrm{R}_{2}\right)$ describes the carbonation of the $\mathrm{C}-\mathrm{S}-\mathrm{H}$ by successive decalcifications, starting from $\mathrm{C}-\mathrm{S}-\mathrm{H}_{1.6}$ and leading to the end product $\mathrm{SiO}_{2}\left(\mathrm{H}_{2} \mathrm{O}\right)_{0.5}$, that is hydrated silica. The precise decalcification process of the $\mathrm{C}-\mathrm{S}-\mathrm{H}$ is not well-known. That is why the reaction pathway of this process is not detailed in this study. Moreover, in order to identify the main chemical reactions during cement paste carbonation, simulations on reactive transport were performed by BRGM using TOUGHREACT, a software wellsuited (see [10]) for modelling multiphase geochemical reactive transport in porous and fractured media. The results (see [11]) show that predominant mechanisms of cement paste carbonation are those related to the last two hydrates and that these carbonation reactions can be considered as sequential, instantaneous and complete, which enables to neglect reaction kinetics. Both reactions are assumed to be sequential because as long as non carbonated portlandite remains in the cement paste, the $\mathrm{pH}$ is maintained to a high level $(\mathrm{pH}=13)$ and $\mathrm{CO}_{2}$ can not attack $\mathrm{C}-\mathrm{S}-\mathrm{H}$. Nevertheless, as soon as Portlandite has been fully carbonated, the $\mathrm{pH}$ decreases and $\mathrm{CO}_{2}$ can carbonate the $\mathrm{C}-\mathrm{S}-\mathrm{H}$ (see [2]). The assumption of instantaneous reactions is made because relative to the slow diffusion of ions in the in-pore fluid, the characteristic times of the chemical reactions are very small. Generally, we can write the carbonation reactions as:

$$
\mathrm{a}_{1, \mathrm{Ri}} \mathrm{M}_{1, \mathrm{Ri}}^{\mathrm{S}}+\mathrm{CO}_{2} \rightarrow \underline{\mathrm{CaCO}_{3}}+\mathrm{a}_{\mathrm{H}_{2} \mathrm{O}, \mathrm{Ri}} \mathrm{H}_{2} \mathrm{O}+\mathrm{a}_{2, \mathrm{Ri}} \mathrm{M}_{2, \mathrm{Ri}}^{\mathrm{S}}
$$


where $a_{j, R i}$ is the stoechiometric coefficient of the solid mineral $M_{j, R i}^{S_{j}}$ in the reaction $\left(R_{i}\right)$, with $j=1$ for a reactant and $\mathrm{j}=2$ for a product in the same reaction. The arrow under calcite symbolizes the precipitation of the mineral.

Finally, the reaction pathway considered here corresponds to the two reactions below:

$$
\begin{gathered}
\mathrm{CH}+\mathrm{CO}_{2} \rightarrow \underline{\mathrm{CaCO}_{3}}+\mathrm{H}_{2} \mathrm{O} \\
0.625 \mathrm{C}-\mathrm{S}-\mathrm{H}_{1.6}+\mathrm{CO}_{2} \rightarrow \underline{\mathrm{CaCO}_{3}}+1.3 \mathrm{H}_{2} \mathrm{O}+0.625 \mathrm{SiO}_{2}\left(\mathrm{H}_{2} \mathrm{O}\right)_{0.5}
\end{gathered}
$$

From these chemical reactions, we assume that the reactive constituents of the cement paste relatively to the carbonation process are only $\mathrm{CH}$ and $\mathrm{C}-\mathrm{S}-\mathrm{H}$. Moreover we consider that, in spite of the presence of chemically inert constituents, the solid phase S of the porous media is composed of several minerals $\mathrm{M}_{\mathrm{i}}$ and calcite $\mathrm{C}$ such as: $\mathrm{S}=\left\{\mathrm{M}_{\mathrm{i}}, \mathrm{C}\right\}=\left\{\mathrm{CH}, \mathrm{C}-\mathrm{S}-\mathrm{H}, \mathrm{SiO}_{2}\left(\mathrm{H}_{2} \mathrm{O}\right)_{0.5}, \mathrm{CaCO}_{3}\right\}$, where $\mathrm{C}-\mathrm{S}-\mathrm{H}$ can have several densities depending on the decalcification stage. Concerning the fluid phase F, it contains water and dissolved species due to the dissolution of $\mathrm{CO}_{2}$ and of the solid minerals: $\mathrm{F}=\{w, \alpha\}=\left\{\mathrm{H}_{2} \mathrm{O}\right.$, $\left.\mathrm{HCO}_{3}{ }^{-}, \mathrm{CO}_{3}{ }^{2-} \mathrm{Ca}^{2+}, \mathrm{OH}^{-}, \ldots\right\}$. These notations will be useful in the thermodynamic analysis.

1.2 Cement paste as a saturated poroelastic medium

In this part, the mechanical behaviour of saturated cement paste is studied under the framework of non equilibrium thermodynamics. Indeed, as demonstrated by [12] and [13], such a framework is relevant because it allows the extension to porous media of the classic balance equations of continuum mechanics relative to mass, momentum, energy and entropy. The key points of this approach are recalled in the following paragraphs.

Let $n_{i} \times d \Omega_{0}$ be the number of moles of the species $i$ present in an infinitesimal representative volume of the porous medium $d \Omega_{t}$ (i.e. $n_{i}$ is the apparent molar density of species $i$ ) in the initial state. The isothermal Gibbs-Duhem equation assuming chemical equilibrium can be written relatively to the in-pore solution and to the carbonate crystal so that: 


$$
\begin{aligned}
& -\delta_{\mathrm{C}} \frac{\mathrm{d} p_{\mathrm{C}}}{\mathrm{d} t}+n_{\mathrm{C}}^{\mathrm{S}} \frac{\mathrm{d} \mu_{\mathrm{C}}^{\mathrm{S}}}{\mathrm{d} t}=0 \\
& -\varphi_{\mathrm{F}} \frac{\mathrm{d} p_{\mathrm{F}}}{\mathrm{d} t}+\sum_{\mathrm{i} \in \mathrm{F}} n_{\mathrm{i}} \frac{\mathrm{d} \mu_{\mathrm{i}}}{\mathrm{d} t}=0
\end{aligned}
$$

where $p_{\mathrm{C}}$ and $p_{\mathrm{F}}$ are the pressures of the carbonate crystal and the in-pore fluid respectively and $\mu_{\mathrm{C}}^{\mathrm{S}}$ and $\mu_{\mathrm{i} \in \mathrm{F}}$ stand for the molar chemical potentials of calcite and aqueous species $i$ of the fluid phase respectively. In Eq. (2), the energy transmitted through the shear stress is neglected compared to that transmitted through the normal stress between carbonate crystals and solid matrix.

Let $\phi_{0}$ be the initial porosity so that the initial pore volume inside $d \Omega_{0}$ is $\phi_{0} \times d \Omega_{0}$. A distinction is made between the effective porosity and the overall porosity of the porous medium. The former, denoted $\phi_{F}$, corresponds to the pore volume occupied by the in-pore fluid per unit volume of porous medium. The latter, denoted by $\phi$, corresponds to the pore space per unit of initial volume of porous medium which is not occupied by the solid phase. The difference between these two porosities is denoted by $\delta_{C}$. By definition, the volume $\delta_{C} \times d \Omega_{0}$ refers to the pore volume occupied by carbonate crystals inside $d \Omega_{t}$. This distinction is important in order to follow precisely the processes of dissolution and precipitation of the cement matrix and the calcite. These porosities are linked by the following relation:

$$
\phi=\phi_{\mathrm{F}}+\delta_{\mathrm{C}}
$$

When a cementitious material is submitted to the presence of $\mathrm{CO}_{2}$, it undergoes several variations on porosities. Some are due to chemical reactions, denoted $\phi_{L}$ for leaching of cement matrix and $\phi_{P}$ for calcite precipitation. The other variations of porosity are related to the deformation of the porous medium with $\varphi_{F}$ and $\varphi_{C}$ the deformation of the porosity filled respectively by fluid phase and by calcite phase. The porosities involved in Eq. (3) can thus be rewritten as follows:

$$
\begin{aligned}
& \phi_{\mathrm{F}}=\phi_{0}+\phi_{\mathrm{L}}-\phi_{\mathrm{P}}+\varphi_{\mathrm{F}} \\
& \delta_{\mathrm{C}}=\phi_{\mathrm{P}}+\varphi_{\mathrm{C}}
\end{aligned}
$$

Let $\boldsymbol{\varepsilon}$ be the overall infinitesimal strain tensor of the representative elementary volume and $\boldsymbol{\sigma}$ the overall stress tensor to which the considered system is subjected. $d \Omega_{t}$ being an open thermodynamic system, it will exchange during its evolution moles of species $\alpha$ with its surroundings. Finally, let us 
introduce $\psi$ the free energy of the system. The first and second laws of thermodynamics combine to provide the isothermal Clausius-Duhem inequality related to the system in the form (see [12]):

$$
\boldsymbol{\sigma}: \frac{\mathrm{d} \boldsymbol{\varepsilon}}{\mathrm{d} t}-\sum_{\mathrm{i} \in \mathrm{F}} \mu_{i} \operatorname{div} \mathbf{w}_{i}-\frac{\mathrm{d} \psi}{\mathrm{d} t} \geq 0
$$

where $\mathbf{w}_{\mathrm{i} \in \mathrm{F}}$ is the vector of molar transport of the aqueous species $i$. Thus $-\operatorname{div} \mathbf{w}_{\mathrm{i} \in \mathrm{F}} d \Omega_{t}$ is the rate of moles of aqueous species $i$ externally supplied to the infinitesimal porous element $d \Omega_{t}$ by its contiguous elements. Considering Fick's diffusion law and Darcy's law for the diffusive and advective transport of species $i$, the vector $\mathbf{w}_{\mathrm{i} \in \mathrm{F}}$ can be expressed as:

$$
\mathbf{w}_{\mathrm{i} \in \mathrm{F}}=-d_{\mathrm{eff}} \operatorname{grad}\left(\frac{n_{\mathrm{i} \in \mathrm{F}}}{\phi_{\mathrm{F}}}\right)-n_{\mathrm{i} \in \mathrm{F}} \frac{\kappa}{\eta_{\mathrm{vis}}} \operatorname{grad} p_{\mathrm{F}}
$$

with $d_{\text {eff }}$ the effective diffusion coefficient, $\kappa$ the intrinsic permeability, $\eta_{\text {vis }}$ the dynamic viscosity of the fluid phase and $n_{\mathrm{i} \in \mathrm{F}}$ the number of moles of the species $i$ per unit of overall volume. From Gibbs-Duhem equalities (1) and (2) and Clausius-Duhem inequality (5), assuming isothermal conditions, the constitutive equations of isotropic linear poroelasticity can be deduced (see [12] for more details):

$$
\begin{gathered}
\boldsymbol{\sigma}-\boldsymbol{\sigma}_{\mathbf{0}}=\left(K-\frac{2}{3} G\right)\left(\varepsilon-\varepsilon_{0}\right) \mathbf{1}+2 G\left(\boldsymbol{\varepsilon}-\boldsymbol{\varepsilon}_{\mathbf{0}}\right)-\sum_{\mathrm{K}=\mathrm{F}, \mathrm{C}} b_{\mathrm{K}}\left(p_{\mathrm{K}}-p_{\mathrm{K}, 0}\right) \mathbf{1} \\
\varphi_{\mathrm{J}}-\varphi_{\mathrm{J}, 0}=b_{\mathrm{J}}\left(\varepsilon-\varepsilon_{0}\right)+\sum_{\mathrm{K}=\mathrm{F}, \mathrm{C}} \frac{p_{\mathrm{K}}-p_{\mathrm{K}, 0}}{N_{\mathrm{JK}}} ; J=F, C
\end{gathered}
$$

In equations (7) and (8), $\varepsilon=\operatorname{tr}(\varepsilon)$ is the volumetric deformation, $K$ and $G$ are respectively the bulk modulus and the shear modulus of the empty porous solid while $b_{J}$ and $N_{J K}$ are respectively the generalised Biot coefficients and the generalised poroelastic coupling moduli.

Finally, to evaluate the failure risk of the well-bore cement, the classic Drucker-Prager criterion will be used a posteriori that is at the end of the poroelastic computation:

$$
3 \alpha \sigma+\beta \sqrt{J_{2}}-k \leq 0 \quad \text { with } \alpha=\frac{\left|R_{c}\right|-R_{t}}{\left|R_{c}\right|+R_{t}}, \beta=1 \text {, and } k=2 \frac{\left|R_{c}\right| \times R_{t}}{\left|R_{c}\right|+R_{t}}
$$

where $\sigma=\operatorname{tr}(\boldsymbol{\sigma}) / 3$ denotes the mean stress, $J_{2}$ the second invariant of the deviatoric stress while $R_{c}$ and $R_{t}$ are respectively the compressive strength and the tensile strength of the material studied.

The chemical reactions accounted for in this study will induce changes of porosity and of the mineral 
composition of the solid phase. A micromechanical approach will be used to assess these effects on the poroelastic properties of the porous medium. The details of these calculations are developed in the second part of this paper.

\subsection{Chemo-poromechanical model}

To highlight the coupling between the chemical reactions and the poromechanical behaviour, we express the overall mass conservation of the in-pore fluid in the following form:

$$
\frac{1}{\rho_{\mathrm{F}}} \frac{\mathrm{d}\left(\phi_{\mathrm{F}} \rho_{\mathrm{F}}\right)}{\mathrm{d} t}=\frac{1}{\rho_{\mathrm{F}}} \sum_{\mathrm{i} \in \mathrm{F}} \mathrm{M}_{\mathrm{i}} \frac{\mathrm{d} n_{\mathrm{i}}}{\mathrm{d} t}
$$

Equation (10) simply expresses the fact that any variation in the total mass of in-pore fluid per unit of overall initial volume must be linked to the variations of the number of moles of the species it contains. Here, $\mathrm{M}_{\mathrm{i}}$ and $\mathrm{d} n_{\mathrm{i} \in \mathrm{F}} / \mathrm{d}$ tare respectively the molar mass and the temporal variation of the apparent molar concentration of a fluid species $i$ due to the molar transport of $\mathrm{CO}_{2}$ as well as the chemical reactions occurring in the cement paste.

$$
\frac{\mathrm{d} n_{\mathrm{i} \in \mathrm{F}}}{\mathrm{d} t}=-\operatorname{div} \mathbf{w}_{\mathrm{i} \in \mathrm{F}}+\stackrel{\circ}{n}_{\mathrm{i} \in \mathrm{F}}
$$

Similarly, the mass balance for a particular solid mineral, assuming that its molar transport is not significant, can be expressed as:

$$
\frac{\mathrm{d} n_{\mathrm{Mi}}^{\mathrm{S}}}{\mathrm{d} t}={ }_{\circ \mathrm{S}}^{\mathrm{S}} \quad \text { and } \quad \frac{\mathrm{d} n_{\mathrm{C}}^{\mathrm{S}}}{\mathrm{d} t}={ }^{\circ} n_{\mathrm{C}}
$$

with $\stackrel{\circ}{n_{\mathrm{i} \in \mathrm{F}}}, \stackrel{\circ}{n_{\mathrm{Mi}}}$ and $\stackrel{\circ}{n_{\mathrm{C}}}$ the molar variations of dissolved species, solid minerals and carbonate crystals due to chemical reactions and $\mathbf{w}_{\mathrm{i} \in \mathrm{F}}$ the molar transport (see Eq. (6)).

We introduce the bulk modulus $K_{F}$ of the in-pore fluid and the bulk modulus $K_{C}$ of the carbonate crystals, $\rho_{C}$ its density and $p_{C}$ its pressure. $\gamma_{i}$ represents the fluid density variation induced by the chemical composition changes. Under the assumption of no transmission of shear between calcite and cement 
matrix (see Eq. (2)), the variation of density of the fluid and the calcite under isothermal conditions can be written in the form:

$$
\begin{gathered}
\frac{1}{\rho_{\mathrm{F}}} \frac{\mathrm{d} \rho_{\mathrm{F}}}{\mathrm{d} t}=\frac{1}{K_{\mathrm{F}}} \frac{\mathrm{d} p_{\mathrm{F}}}{\mathrm{d} t}+\sum_{i \in F} \gamma_{i} \frac{\mathrm{d} n_{i}}{\mathrm{~d} t} \text { with } \gamma_{i \in F}=\left[\rho_{F} \frac{d\left(1 / \rho_{F}\right)}{d n_{i}}\right]_{p_{F}, n_{j}, j \neq i} \\
\frac{1}{\rho_{\mathrm{C}}} \frac{\mathrm{d} \rho_{\mathrm{C}}}{\mathrm{d} t}=\frac{1}{K_{\mathrm{C}}} \frac{\mathrm{d} p_{\mathrm{C}}}{\mathrm{d} t}
\end{gathered}
$$

Moreover, the reaction of precipitation of calcium carbonates is assumed to be instantaneous because the kinetics of dissolution/precipitation is much faster than the diffusive transport of $\mathrm{CO}_{2}$ in the liquid phase (cf [14]). This amounts to say that the thermodynamic equilibrium between dissolved species and carbonate crystals holds true at any time and in each infinitesimal representative volume. In other words, the chemical potential of carbonate crystals is equal to the sum of that of the dissolved species:

$$
\mu_{\mathrm{C}}=\mu_{\mathrm{Ca}^{2+}}+\mu_{\mathrm{CO}_{3}^{2-}}
$$

Thanks to Eq. (4), (10), (11) and (13) and assuming that reactions of precipitation occur under constant energy, the conservation of fluid mass can be expressed as follows:

$$
\frac{\phi_{\mathrm{F}}}{K_{\mathrm{F}}} \frac{\mathrm{d} p_{\mathrm{F}}}{\mathrm{d} t}+\frac{\mathrm{d} \varphi_{\mathrm{F}}}{\mathrm{d} t}=\sum_{\mathrm{R}_{\mathrm{i}}}\left(v_{\mathrm{C}}^{\mathrm{S}}+a_{2, R_{i}} v_{\mathrm{M}_{2}, R_{i}}^{\mathrm{S}}-a_{1, R_{i}} v_{\mathrm{M}_{1}, R_{i}}^{\mathrm{S}}+a_{\mathrm{H}_{2} \mathrm{O}, R_{i}} v_{\mathrm{H}_{2} \mathrm{O}, R_{i}}^{\mathrm{F}}\right) \stackrel{\circ}{\xi_{R_{i}}}+\operatorname{div}\left(\frac{\kappa}{\eta_{\text {vis }}} \operatorname{grad} p_{\mathrm{F}}\right)
$$

where $a_{j, R i}$ is the stoechiometric coefficient of the solid mineral $M_{j, R i}^{S}$ in the reaction $\left(R_{i}\right)$, with $j=1$ for a reactant and $\mathrm{j}=2$ for a product in the same reaction and $v_{\mathrm{k}}$ is the molar volume of the calcite or the solid mineral $\mathrm{M}_{\mathrm{j}, \mathrm{Ri}}^{\mathrm{S}}$ or water produced by the reaction $\left(\mathrm{R}_{\mathrm{i}}\right)$.

In equation (16) four contributions to the variation of pore fluid pressure are distinguished. Indeed, an increase of in-pore fluid pressure can come from first a net fluid influx represented by the last term (Darcy's law), second a variation of pore volumes due to lixiviation or to precipitation and finally from a pore deformation (term $\left.\frac{\mathrm{d} \varphi_{\mathrm{F}}}{\mathrm{d} t}\right)$.

According to the assumptions related to reactions kinetics previously introduced, the advancement rate $\xi_{\mathrm{Ri}}^{\circ}$ of each reaction $\mathrm{R}_{\mathrm{i}}$ can directly be estimated from the quantity of $\mathrm{CO}_{2}$ supplied to the system by diffusion and advection: 


$$
\left\{\begin{array}{l}
\left(\xi_{\mathrm{R}_{2}}^{\circ}=0, \xi_{\mathrm{R}_{1}}^{\circ}=X_{R}\right) \text { if } \mathrm{M}_{1, \mathrm{R}_{1}}^{\mathrm{S}}>0 \\
\left(\xi_{\mathrm{R}_{2}}^{\circ}=X_{R}, \xi_{\mathrm{R}_{1}}^{\circ}=0\right) \text { if } \mathrm{M}_{1, \mathrm{R}_{1}}^{\mathrm{S}}=0 \text { and } \mathrm{M}_{1, \mathrm{R}_{2}}^{\mathrm{S}}>0 \\
\left(\xi_{\mathrm{R}_{2}}^{\circ}=0, \xi_{\mathrm{R}_{1}}^{\circ}=0\right) \text { if } \mathrm{M}_{1, \mathrm{R}_{1}}^{\mathrm{S}}=0 \text { and } \mathrm{M}_{1, \mathrm{R}_{2}}^{\mathrm{S}}=0 \\
\text { with } X_{R}=\operatorname{div}\left(d_{\mathrm{eff}} \operatorname{grad}\left(\frac{n_{\mathrm{CO}_{2}}}{\phi_{\mathrm{F}}}\right)+n_{\mathrm{CO}_{2}} \frac{\kappa}{\eta_{\text {vis }}} \operatorname{grad} p_{\mathrm{F}}\right)
\end{array}\right.
$$

Finally, the variation of the molar quantity of $\mathrm{CO}_{2}$ corresponds to the quantity brought by advection and diffusion minus the quantity consumed by chemical reactions:

$$
\frac{\mathrm{d} n_{\mathrm{CO}_{2}}}{\mathrm{~d} t}=\operatorname{div}\left(d_{\mathrm{eff}} \operatorname{grad}\left(\frac{n_{\mathrm{CO}_{2}}}{\phi_{\mathrm{F}}}\right)+n_{\mathrm{CO}_{2}} \frac{\kappa}{\eta_{\mathrm{vis}}} \operatorname{grad} p_{\mathrm{F}}\right)-\sum_{\mathrm{R}_{\mathrm{i}}} \xi_{\mathrm{Ri}}
$$

Equation (7) together with the momentum balance condition and equations (16), (17) and (18) define the chemo-poromechanical model. Its implementation into a finite volume code is described in the following section.

\section{NUMERICAL RESOLUTION}

To solve the chemo-poromechanical problem, the proposed model has been implemented into an in-house research code. Poromechanical validation had been made for simple cases with no chemical couplings on the basis of analytical solutions. The cement paste studied here corresponds to a class $\mathrm{G}$ cement with a water-to-cement ratio equal to 0.44 . This value is representative of cement pastes used by the petroleum industry for cementing production wells (see [15] and [16]). In this second part, we first expose the method used to estimate the poro-elastic moduli of the cement paste during the carbonation process. Then the calculation of the other parameters of the model is made explicit. An overview of the main principles of the numerical code is finally exposed.

2.1. Homogenization of poroelastic properties of the porous cement paste

This part deals with the calculation by a homogenization technique of the poroelastic moduli of the 
cement paste during the dissolution/precipitation processes induced by its carbonation. Resorting to upscaling methods is suitable in this study because the chemical reactions induce significant changes of the microstructure of the solid skeleton (dissolution/precipitation phenomena). Since we are interested in the mechanical behaviour of the geomaterials, this approach will provide a physically sound assessment of the chemically-induced alterations of the elastic properties of the cement paste.

The theoretical framework of the homogenization method used in this study is that presented by [17]. In [17], [18], [16] and [19], a micromechanics analysis of the cement paste and cement mortar is performed to predict the properties of these materials with a given micro and macro-structure. A micro-structural analysis of the $\mathrm{C}-\mathrm{S}-\mathrm{H}$ accounting for their high or low densities is developed. Nevertheless, in the present study, a simplified approach is adopted and only the macro-structure of the cement paste (including solely the capillary porosity) is considered.

Here, all components (including $\mathrm{CH}, \mathrm{C}-\mathrm{S}-\mathrm{H}$, calcite, $\mathrm{SiO}_{2}$ and chemically inert phases) have to be considered in the homogenization analysis as regard to their mechanical contribution to the overall stiffness. A mineralogical study has been carried out at the geochemistry laboratory of INPL (Nancy, France) on healthy cement paste samples. This study provides the values of the volumetric proportions of each mineral before carbonation. These values are presented in Table 1 together with the corresponding bulk and shear moduli found in the literature.

The principle of the homogenization technique is to derive the properties of a fictitious homogeneous media which behaves globally the same way as the real heterogeneous body accounting for the pertinent physics at a lower scale. It is then possible to replace this complex heterogeneous media by the fictitious homogenized media. An Eshelbian morphology for the cement paste (see Fig. 1) is assumed, that is to say a spherical inclusion embedded in a reference media. We also consider the isotropy of the local and reference media. A self-consistent scheme is chosen for the homogenization step. The reference medium is consequently the homogenized medium (i.e. the fictitious homogenized medium). The self-consistent scheme is suitable for material whose phases are dispersed in the considered reference volume and when none of these phases plays any specific morphological role. Under these assumptions, we can estimate the poroelastic parameters (bulk and shear moduli, Biot coefficient and Biot skeleton modulus) of the cement 
paste as a homogenized medium by using the simplified following relations:

$$
\begin{gathered}
K_{d}^{\mathrm{hom}}=\sum_{r} f_{r} k_{r} A_{r}^{v} \quad ; \quad G_{d}^{\mathrm{hom}}=\sum_{r} f_{r} g_{r} A_{r}^{d} \\
b^{\mathrm{hom}}=1-\sum_{r} f_{r} A_{r}^{v} \quad ; \quad \frac{1}{N^{\mathrm{hom}}}=\sum_{r} f_{r} \frac{1-A_{r}^{v}}{k_{r}}
\end{gathered}
$$

In the equations (19) and (20), $f_{r}$ is the volumetric proportion of the phase $r, k_{r}$ and $g_{r}$ are elastic parameters of the solids $r$ and $A_{r}^{v}$ and $A_{r}^{d}$ are the volumetric and deviatoric strain localization coefficients (see [16], [17] and [20] for more details).

Thanks to this homogenization method and a local iterative process included in the numerical code, poroelastic parameters of the cement paste can be estimated at any time during the carbonation process.

\subsection{Main parameters and numerical resolution}

To simulate a cement wellbore drilled into a deep reservoir at a post- $\mathrm{CO}_{2}$-injection stage, the chemoporomechanical model with appropriate boundary conditions has been implemented in a finite volume code. At any time, the porous medium is saturated by a single liquid phase, possibly containing dissolved $\mathrm{CO}_{2}$.

In this study, a simplified geometry is considered by assuming axisymmetric conditions under plane strains in the axial direction. Thus the different unknowns only depend on the radial space variable (distance to the well axis) and time. Under the assumption of an isotropic material, the strain and stress tensors are diagonal. According to [21] the effects of porosity changes on the intrinsic permeability $\kappa$ (in $\mathrm{m}^{2}$ ) of this type of cement paste can be captured through the Eq. (21):

$$
\kappa=1.2\left(\frac{\phi_{\mathrm{F}}}{0.26}\right)^{11} \cdot 10^{-19}
$$

The diffusion coefficient $d_{\text {eff }}\left(\right.$ in $\left.\mathrm{m}^{2} \cdot \mathrm{s}^{-1}\right)$ is also assumed [14] to be a function of the porosity and is defined by:

$$
d_{\text {eff }}=100 . \exp \left(9.95 \phi_{\mathrm{F}}-29.08\right)
$$


Values used in this study for the initial porosity, the bulk modulus of the pore fluid, the molar volumes of minerals and water and the parameters used for the Drucker-Prager criterion are summarised in Table 2 and 3.

Concerning the initial mineral composition, the hydrated cement paste considered here is composed of $20 \%$ of Portlandite, $27 \%$ of $\mathrm{C}-\mathrm{S}-\mathrm{H}_{1.6}$ and $25 \%$ of inert components such as aluminates (volume fractions) with an initial porosity of $28 \%$. It should be noted that the calcite phase that precipitates during the carbonation process may eventually dissolve, depending on the $\mathrm{CO}_{2}$ concentration and $\mathrm{pH}$ value (see [7]). Nevertheless and according to experimental observations reported by [3], we do not take into account this possibility in the short-term simulations presented in this study.

The choice of a finite volume code is relevant in the case of reactive transport problems and dissolution/precipitation phenomena. Experiments performed by [3] and [5] show a quite steep separation between the carbonated and healthy parts of the cement paste samples exposed to a $\mathrm{CO}_{2}$-rich fluid. Moreover this front remains sharp during the carbonation process. As a consequence, it can reasonably be assumed that a sharp carbonation front exists at any time in the sample. According to [14] and [22], finite volume spatial discretisation is particularly appropriate for this kind of reactive transport problems because it ensures the mass conservation between each control volume and the numerical scheme stays relatively stable despite the sharp dissolution/precipitation fronts, without numerical instability or convergence problems. In the finite volume method, the modelled domain is discretised into a given number of control volumes associated to central nodes and interfaces. We choose to keep a constant radial step between nodes of volumes and thus between interfaces for the cement part whereas for the caprock domain (present in order to fix realistic mechanical boundary conditions) a geometrical progression is used for the space discretisation. This choice leads to divide the domain of the cement paste in 100 control volumes and in 49 volumes for the caprock part. The unknowns of the problem are the fluid pressure, the advancement rate of reaction, the $\mathrm{CO}_{2}$ concentration and the displacements. Their values are computed at the nodes (and are thus constant per control volume). To fix the boundary conditions, two half-control volumes are added at the boundaries of the structure. The physical domain modelled includes the cement layer of the well and the surrounding caprock which is assumed here chemically inert. The 
metallic casing is not explicitly modelled. Only its effects are accounted for by imposing adequate hydromechanical boundary conditions (see section 3). In our in-house code, an implicit Euler scheme is used for the time discretisation. The Newton-Raphson method is used to solve numerically the problem. Furthermore, with the hypothesis of the sharp carbonation front, we impose that for a given control volume, any incoming $\mathrm{CO}_{2}$ must react with all the reactants in presence before the excess amount can enter the next control volume.

Finally, we assume that the carbonate minerals precipitate on pore walls of the cement matrix and stick to the solid matrix. Under this assumption interfacial effects can be neglected in the mechanical equilibrium between calcite and the pore fluid so that $p_{\mathrm{C}}=p_{\mathrm{F}}$.

\section{RESULTS AND DISCUSSION}

\subsection{Statement of the problem}

Initial and boundary conditions are now made explicit. The cement well is modelled as a cylindrical domain with an internal radius of $0.089 \mathrm{~m}$, an external radius of $0.108 \mathrm{~m}$ and a unit height. We are interested in the effects of a leakage of gas by dissolution in water at the interface between the caprock and the cement well seen as a preferential path (see [23] and [24]). Hence, $\mathrm{CO}_{2}$ will be assumed to enter the cement paste at its outer surface and migrates towards the inner surface. We consider a uniform fluid pressure of $10 \mathrm{MPa}$ at the beginning, which roughly corresponds to the fluid pressure at around $1000 \mathrm{~m}$ depth in the post-injection stage.

The focus of our analysis being the effects and consequences of the chemo-mechanical couplings inside the cement paste, the caprock is simply modelled as a chemically inert, poro-elastic material. Before drilling the well, the caprock is assumed to be in a state of homogeneous and isotropic stress of 25 $\mathrm{MPa}$. To take into account the well drilling and the well cementation, a partial deconfinement of $50 \%$ at the inner surface of the cement well is assumed. This new stress field becomes the reference state for the calculation of the displacements. At this new initial state, null displacement $\left(u_{\mathrm{r}}=0\right)$ and strains $\left(\varepsilon_{\mathrm{r}}=\varepsilon_{\theta}=0\right)$ are supposed at all material points under the assumption of non-shrinking and impermeable 
casing.

In addition, the cement paste is initially intact and does not contain any carbonate mineral before the injection of $\mathrm{CO}_{2}$. The in-pore water does not contain any dissolved $\mathrm{CO}_{2}$ and is subjected to a uniform pressure of $10 \mathrm{MPa}$ at the initial state. To model a $\mathrm{CO}_{2}$-rich fluid leakage between the caprock and the cement paste, we assume at all time drained conditions at this interface. A fluid pressure of $10 \mathrm{MPa}$ is thus imposed in this region. With the arrival of $\mathrm{CO}_{2}$-rich fluid, a carbon dioxide concentration corresponding to the saturated concentration of $\mathrm{CO}_{2}$ in water is also fixed. At the inner surface of the cement paste, in order to account for the metallic casing, an impermeable boundary is assumed (no flow of any species). Finally, concerning the mechanical problem, we choose to apply a constant compressive radial stress of $25 \mathrm{MPa}$ at the external surface of the caprock (representing a boundary condition far from the well) and null displacements on the internal surface of the cement well. Figure 2 summarizes boundary conditions imposed on the studied structure.

\subsection{Results}

Selected numerical results are now presented. The behaviour of the caprock and the cement well during 180 days has been computed. Only the results concerning the cement part are presented here. An influx of $\mathrm{CO}_{2}$ is imposed at the cement-caprock interface (at $r=r_{\mathrm{ext}}=0.108 \mathrm{~m}$ ). Figure 3 shows the spatial distribution of the volumetric proportions of each mineral species after 10 days of exposure and 40 days of exposure. In the figure, "other minerals" refer to the aluminates which are chemically inert and the hydrated silicates which are formed after the complete decalcification of the $\mathrm{C}-\mathrm{S}-\mathrm{H}$ by the carbonation process. After 10 days of exposure to $\mathrm{CO}_{2}$-rich fluid, $6.4 \mathrm{~mm}$ of the sample are fully carbonated. Such a carbonation depth is realistic in front of the experimental results of [3]. The carbonated zone, located between $\mathrm{r}=0.1080 \mathrm{~m}$ and $\mathrm{r}=0.1016 \mathrm{~m}$ does not contain $\mathrm{CH}$ and $\mathrm{C}-\mathrm{S}-\mathrm{H}$ anymore and is only composed of calcite, hydrated amorphous silica and the other inert components. In the adjacent volume between $\mathrm{r}=0.1016 \mathrm{~m}$ and $\mathrm{r}=0.1004 \mathrm{~m}, \mathrm{CH}$ and $\mathrm{C}-\mathrm{S}-\mathrm{H}$ are disappearing whereas calcite and silica are being generated. The dissolution/precipitation front is located inside this volume. By comparing Fig. 3(a) and Fig. 3(b), we can observe the advancement of the carbonation front through the well and that this front, in 
consistency with our assumptions, remains sharp. From Fig. 4, the cement well will be fully carbonated after 70 days of carbon exposure. These modifications of composition of the cement matrix induce evolutions of its porosity. Figure 5 shows how the chemical reactions affect the fluid porosity $\phi_{\mathrm{F}}$ of the cement paste during the carbonation. As a consequence of the incoming of $\mathrm{CO}_{2}$, the cement matrix is partially dissolved, followed by the precipitation of new minerals. Thus, the supply of $\mathrm{CO}_{2}$ mass and the formation of calcite and $\mathrm{SiO}_{2}$ decrease the porosity of the cement paste, from $28 \%$ to $24.1 \%$. The loss of porosity is also observable in Fig. 3 where we can see that new minerals occupy more space than $\mathrm{CH}$ and $\mathrm{C}-\mathrm{S}-\mathrm{H}$. This reduction of porosity leads to, as seen from equations (21) and (22), an alteration of the transport properties of the cement paste such as an effective decrease of the permeability which is divided by 5 (see Fig. 6) and of the diffusivity coefficient of the $\mathrm{CO}_{2}$ in water which is divided by 1.5 in altered zones. Furthermore, as a consequence of the decrease of the fluid porosity due to formation of carbonate minerals, fluid pressure increases and cannot be immediately dissipated because of the reduced permeability of the cement paste.

From Fig. 7, we can see an interior zone of higher fluid pressure bounded by the carbonation front. This overpressure has its origin in the impermeable boundary conditions imposed at the inner surface (casing) and in the carbonation mechanism because carbonation reaction produces water (see Eq. $\mathrm{R}_{1}$ and $\mathrm{R}_{2}$ ) whose pressure can not be instantaneously dissipated because of the decrease of porosity and permeability. The associated pressure gradient induces a water flow directed towards the external surface of the cement well. These results emphasize the couplings between transport phenomena and chemical processes.

Additionally, the carbonation mechanisms involve also the alteration of the mechanical properties. Indeed, as can be seen in Fig. 8, the bulk modulus is divided by 1.5 , from $7.7 \mathrm{GPa}$ to $5.3 \mathrm{GPa}$, and the shear modulus, initially equal to $3.7 \mathrm{GPa}$ is reduced to about $2.8 \mathrm{GPa}$ at the final state. The cement matrix is consequently degraded. This highlights a potential risk for the durability and safety of $\mathrm{CO}_{2}$ storage.

Finally, in spite of the alteration of the elastic moduli, radial displacements are low (about $6.10^{-5} \mathrm{~mm}$ at the external surface of the cement) (see Fig. 9). It can be seen that the carbonation process leads to a small swelling of the cement well under the prescribed boundary conditions. Figure 10 shows the radial dilatation along the cement paste layer and an increase of radial deformations at the carbonation front. 
Figures 11 and 12 show the distribution of radial stress and a posteriori calculated Drucker-Prager yield criterion. For the case under study, taking into account the plastic behaviour of the material is not relevant. It should be noted that in some situations a risk of damage can be expected, as shown by experimental results presented in [3].

To show the influence of initial porosity and transport properties on the risk of damage, simulations with a lower initial porosity and a lower initial ratio $\kappa / d_{\text {eff }}$ were performed.

In the first parametric study, we choose to impose a initial ratio $\kappa / d_{\text {eff }}$ lower than in the reference case with a ratio divided by 10 and an initial porosity of $28 \%$. In this case, we first note that chemical reactions are strongly faster and that the cement paste is fully carbonated after 7 days of $\mathrm{CO}_{2}$ exposure. Moreover, the plateau of the fluid pressure generated by the carbonation process is a little bit higher than in the reference case, with a maximum of $10.6 \mathrm{MPa}$ instead of $10.06 \mathrm{MPa}$ in the reference case. Finally, the yield criterion (see Fig. 13) increases at each carbonation front. These results are explained by the fact that, with a higher initial diffusive coefficient than in the reference case, the propagation of the carbon dioxide in the medium, mainly governed by diffusive transport in our study, is accelerated. Furthermore, an increase of the reaction rate (this latter being limited by the $\mathrm{CO}_{2}$ supply with our assumptions) implies an increase of the production of water. Thus, water produced by chemical reactions needs much time to be evacuated into the porous medium.

In the second parametric study, the initial porosity is fixed to $18 \%$ instead of $28 \%$ in the reference case presented previously. With this low initial porosity, the cement matrix tends to be very altered by the carbonation mechanisms. Fig. 14, representing the fluid pressure generated in the cement matrix at some times, shows that the overpressure increases significantly between the carbonation front and the casing. It is explained by the fact that a decrease of the initial porosity value is accompanied by an increase of the volume of reactive species in the REV and an initial value of $\kappa / d_{\text {eff }}$ (which is linked to porosity) approximately divided by 10 . The carbonation process thus involves an increase of the quantity of produced water, itself produced more rapidly because of the reduce value of the $\kappa / d_{\text {eff }}$ ratio. This overpressure involves a very high increase of the Drucker-Prager criterion values (see Fig. 15) which 
shows that in these conditions, non reversible deformations are expected. In this case, the cement well could be heavily altered.

\section{CONCLUSION}

In this study, a chemo-poromechanical model applied to carbon dioxide exposure of cement wells has been presented. The model has been implemented in an in-house finite volume code to predict the behaviour of the cement paste under a possible carbon dioxide leakage through the interface between the caprock and the cement well. This code solves in a fully coupled manner the chemo-poromechanical problem being studied. A self-consistent scheme has been used to obtain homogenized mechanical properties and to assess the alteration of mechanical moduli by taking into account calcite and other minerals produced during the carbonation process. Transport properties and elastic parameters of the medium are altered by the carbonation process. The carbonation effects are low but we have to keep in mind that the study is under the assumption of local equilibrium. Therefore, high decrease of porosity, high over pressurization or significant decrease of elastic modulus can not be observed in these simulations. But yet, an appreciable amount of porosity decrease and elastic parameters alteration had to be noticed.

To finish with, a parameter study has shown the importance of the choice of the initial parameters and thus that correctly formulated cement pastes have to be used to ensure well integrity. Furthermore, the couplings between the chemical effects, the transport and the mechanics are highlight and show the complexity of the carbon storage issue. To assess the safety of the storage, damage risk through the calculation of a plastic or damage criterion is necessary.

It is worth noting that the modelling framework proposed in the present study could be extended to model the chemo-poromechanical of the reservoir rock and the caprock when subjected to the presence of an acid fluid $\left(\mathrm{CO}_{2}\right.$-rich brine). In such a case, the chemical reactions would have to be updated but the general methodology would still be applicable. 


\section{Acknowledgements}

This work has been supported by French Research National Agency (ANR) through Captage et stockage $\mathrm{du} \mathrm{CO}_{2}$ program (project INTERFACE n_ANR-08-PCO2-006).

The authors wish to thank Frédéric Wertz (BRGM, Orléans, France) for the interesting discussions on his geo-chemical simulations performed using TOUGHREACT.

\section{References}

1. J. W. Carey, M. Wigand, S. J. Chipera, G. WoldeGabriel, R. Pawar, P. C. Lichtner, S. C. Wehner, M. A. Raines, and G. D. \& Jr. Guthrie. Analysis and performance of oil well cement with 30 years of $\mathrm{CO}_{2}$ exposure from the SACROC Unit, West Texas, USA. International Journal of Greenhouse Gas Control, 1 (1): 75 - 85, 2007. ISSN 1750-5836. doi: 10.1016/S1750-5836(06)00004-1. 8th International Conference on Greenhouse Gas Control Technologies - GHGT-8.

2. Andrew Duguid and George W. Scherer. Degradation of oilwell cement due to exposure to carbonated brine. International Journal of Greenhouse Gas Control, 4 (3): 546 - 560, 2010. ISSN 1750 5836. doi: 10.1016/j.ijggc.2009.11.001.

3. A. Fabbri, J. Corvisier, A. Schubnel, F. Brunet, B. Goffé, G. Rimmele, and V. Barlet-Gouédard. Effect of carbonation on the hydro-mechanical properties of portland cements. Cement and Concrete Research, 39 (12): 1156 - 1163, 2009. ISSN 0008-8846. doi: 10.1016/j.cemconres.2009.07.028.

4. E. Bemer and JM Lombard. From injectivity to integrity studies of $\mathrm{CO}_{2}$ geological storage. Oil \& Gas Science and Technology - Revue de l'Institut Français du Pétrole, 65 (3): 445-459, 2009.

5. J. Corvisier, F. Brunet, A. Fabbri, S. Bernard, N. Findling, G. Rimmele, V. Barlet-Gouedard, O. Beyssac, and B. Goffe. Raman mapping and numerical simulation of calcium carbonates distribution in experimentally carbonated portland-cement cores. European Journal of Mineralogy, 22 (1): 63, 2010. 6. M. Thiery. Modélisation de la carbonatation atmosphérique des bétons. $\mathrm{PhD}$ thesis, Thèse de doctorat, École Nationale des Ponts et Chaussées, Paris, 2005. 
7. B. M. Huet, Jean H. Prevost, and George W. Scherer. Quantitative reactive transport modeling of portland cement in co2-saturated water. International Journal of Greenhouse Gas Control, 4 (3): 561 574, 2010. ISSN 1750-5836. doi: DOI: 10.1016/j.ijggc.2009.11.003.

8. A. Fabbri, N. Jacquemet, and D.M. Seyedi. A chemo-poromechanical model of oilwell cement carbonation under $\mathrm{CO}_{2}$ geological storage conditions. Cement and Concrete Research, 42 (1): 8 - 19, 2012. ISSN 0008-8846. doi: $\underline{10.1016 / j . c e m c o n r e s .2011 .07 .002 . ~}$

9. N. Jacquemet, J. Pironon, and J. Saint-Marc. Mineralogical changes of a well cement in various $\mathrm{H}_{2} \mathrm{~S}-\mathrm{CO}_{2}$ (-brine) fluids at high pressure and temperature. Environmental Science and Technology, 42 (1): 282-288, 2008

10. T. Xu, E. Sonnenthal, N. Spycher, and K. Pruess. TOUGHREACT - a simulation program for non-isothermal multiphase reactive geochemical transport in variably saturated geologic media: Applications to geothermal injectivity and $\mathrm{CO}_{2}$ geological sequestration. Computers \& Geosciences, 32 (2): 145 - 165, 2006. ISSN 0098-3004. doi: 10.1016/j.cageo.2005.06.014.

11. Frederic Wertz, Fabrizio Gherardi, Philippe Blanc, Anne-Gaëlle Bader, and Antonin Fabbri. Cement $\mathrm{CO}_{2}$ - alteration propagation at the well-caprock-reservoir interface and influence of diffusion. International Journal of Greenhouse Gas Control, in press, 2013.

12. O. Coussy. Poromechanics. John Wiley \& Sons Inc, 2004.

13. O. Coussy. Mechanics and physics of porous solids. Wiley, 2010.

14. M. Mainguy. Modèles de diffusion non linéaires en milieux poreux. Application à la dissolution et au séchage des matériaux cimentaires. $\mathrm{PhD}$ thesis, Thèse de doctorat, École Nationale des Ponts et Chaussées, Paris, 1999.

15. N. Neuville, E. Lecolier, G. Aouad, A. Rivereau, and D. Damidot. Effect of curing conditions on oilwell cement paste behaviour during leaching: Experimental and modelling approaches. Comptes Rendus Chimie, 12 (3-4): 511-520, 2009.

16. S. Ghabezloo. Association of macroscopic laboratory testing and micromechanics modelling for the evaluation of the poroelastic parameters of a hardened cement paste. Cement and Concrete Research, 40 (8): 1197 - 1210, 2010. ISSN 0008-8846. doi: 10.1016/j.cemconres.2010.03.016. 
17. F. Ulm, G. Constantinides, and F. Heukamp. Is concrete a poromechanics materials? - a multiscale investigation of poroelastic properties. Materials and Structures, 37: 43-58, 2004. ISSN 1359-5997. doi: $\underline{10.1007 / \mathrm{BF} 02481626 .}$

18. Georgios Constantinides and Franz-Josef Ulm. The effect of two types of C-S-H on the elasticity of cement-based materials: Results from nanoindentation and micromechanical modeling. Cement and Concrete Research, 34 (1): 67 - 80, 2004. ISSN 0008-8846. doi: 10.1016/S0008-8846(03)00230-8.

19. S. Ghabezloo. Effect of the variations of clinker composition on the poroelastic properties of hardened class g cement paste. Cement and Concrete Research, 41 (8): 920 - 922, 2011. ISSN 00088846. doi: 10.1016/j.cemconres.2011.03.022.

20. L. Dormieux, D. Kondo, and F.-J. Ulm. Microporomechanics. Wiley, 2006.

21. S. Ghabezloo. Comportement thermo-poro-mécanique d'un ciment pétrolier. $\mathrm{PhD}$ thesis, Thèse de doctorat, École Nationale des Ponts et Chaussées, Paris, 2008.

22. Robert Eymard, Thierry Gallouet, and Raphaele Herbin. Finite volume methods. In P.G. Ciarlet and J.L. Lions, editors, Solution of Equation in $R^{\wedge} n$ (Part 3), Techniques of Scientific Computing (Part 3), volume 7 of Handbook of Numerical Analysis, pages 713 - 1018. Elsevier, 2000. doi: 10.1016/S1570$\underline{8659(00) 07005-8}$.

23. Michael A. Celia, Stefan Bachu, Jan Martin Nordbotten, Sarah E. Gasda, and Helge K. Dahle. Quantitative estimation of $\mathrm{CO}_{2}$ leakage from geological storage: Analytical models, numerical models, and data needs. In E.S. Rubin, D.W. Keith, C.F. Gilboy, M. Wilson, T. Morris, J. Gale, and K. Thambimuthu, editors, Greenhouse Gas Control Technologies 7, pages 663 - 671. Elsevier Science Ltd, Oxford, 2005. ISBN 978-0-08-044704-9. doi: 10.1016/B978-008044704-9/50067-7.

24. K. Damen, A. Faaij, and W. Turkenburg. Health, safety and environmental risks of underground $\mathrm{CO}_{2}$ storage - overview of mechanisms and current knowledge. Climatic Change, 74 (1): 289-318, 2006. ISSN 0165-0009.

25. Sergio Speziale, Fuming Jiang, Zhu Mao, Paulo J.M. Monteiro, Hans-Rudolf Wenk, Thomas S. Duffy, and Frank R. Schilling. Single-crystal elastic constants of natural ettringite. Cement and Concrete Research, 38 (7): 885 - 889, 2008. ISSN 0008-8846. doi: 10.1016/j.cemconres.2008.02.004. 
26. A. Abou-Chakra Guéry, F. Cormery, J.-F. Shao, and D. Kondo. A comparative micromechanical analysis of the effective properties of a geomaterial: Effect of mineralogical compositions. Computers and Geotechnics, 37 (5): 585 - 593, 2010. ISSN 0266-352X. doi: 10.1016/j.compgeo.2010.02.008. 
Table 1: Parameters used in the homogenization model (from [18], [25] and [26])

\begin{tabular}{|c|c|c|c|c|}
\hline \multirow{2}{*}{ Inclusion } & \multicolumn{2}{|c|}{ Volumetric proportion } & Bulk modulus & Shear modulus \\
\cline { 2 - 3 } & notation & initial value & $(\mathrm{GPa})$ & \\
\hline Porosity & $\phi$ & 0.28 & & 14.5 \\
\hline $\mathrm{CH}$ & $\mathrm{f}_{\mathrm{ch}}$ & 0.20 & 33 & 18.4 \\
\hline $\mathrm{CSH}$ & $\mathrm{f}_{\mathrm{csh}}$ & 0.27 & 25 & 3.2 \\
\hline Lixiviated CSH & $\mathrm{f}_{\mathrm{cshl}}$ & 0 & 5.1 & 9.5 \\
\hline Aluminates $($ ettringite) & $\mathrm{f}_{\mathrm{al}}$ & 0.25 & 27 & 37.4 \\
\hline Calcite $\mathrm{CaCO}_{3}$ & $\mathrm{f}_{\mathrm{cc}}$ & 0 & 69 & \\
\hline
\end{tabular}

Table 2: Material parameters

\begin{tabular}{|c|c|c|c|c|c|c|}
\hline$\phi_{0}$ & $d_{0}\left[m^{2} \cdot s^{-1}\right]$ & $\kappa_{0}\left[m^{2}\right]$ & $\begin{array}{c}\eta_{v i s} \\
{[M P a . s]}\end{array}$ & $K_{F}[M P a]$ & $\mathrm{R}_{\mathrm{c}}[M P a]$ & $\mathrm{R}_{\mathrm{t}}[M P a]$ \\
\hline 0.28 & $4.6510^{-10}$ & $5.7910^{-19}$ & $0.510^{-9}$ & 2200 & -45 & 4.5 \\
\hline
\end{tabular}

Table 3: Molar volumes $\left[\mathrm{cm} 3 . \mathrm{mol}^{-1}\right]$

\begin{tabular}{|c|c|c|c|c|}
\hline$v_{\mathrm{CH}}^{\mathrm{S}}$ & $v_{\mathrm{C}-\mathrm{S}-\mathrm{H}_{1.6}}^{\mathrm{S}}$ & $v_{\mathrm{CaCO}_{3}}^{\mathrm{S}}$ & $v_{\mathrm{SiO}_{2}\left(\mathrm{H}_{2} \mathrm{O}_{0.5}\right.}^{\mathrm{S}}$ & $v_{\mathrm{H}_{2} \mathrm{O}}^{\mathrm{F}}$ \\
\hline 33.1 & 84.7 & 36.9 & 31 & 18.25 \\
\hline
\end{tabular}




\section{Illustrations}

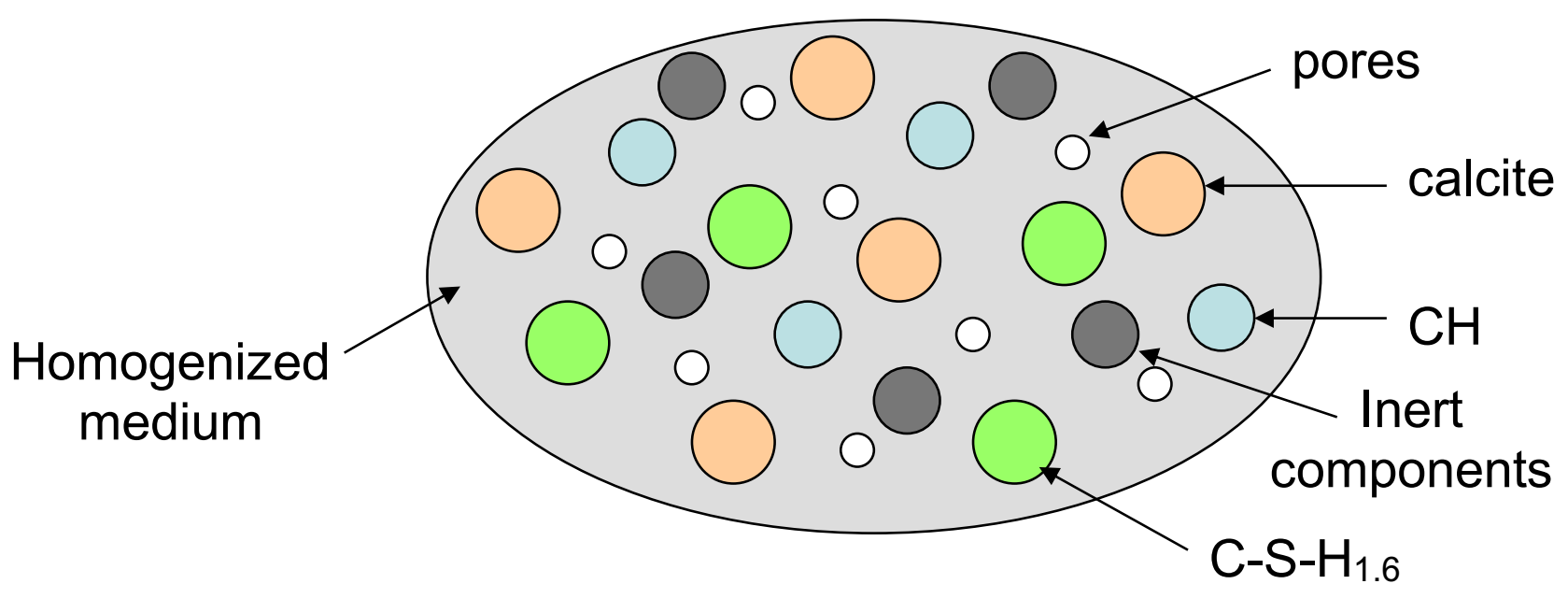

Figure 1: Representation of the cement paste REV

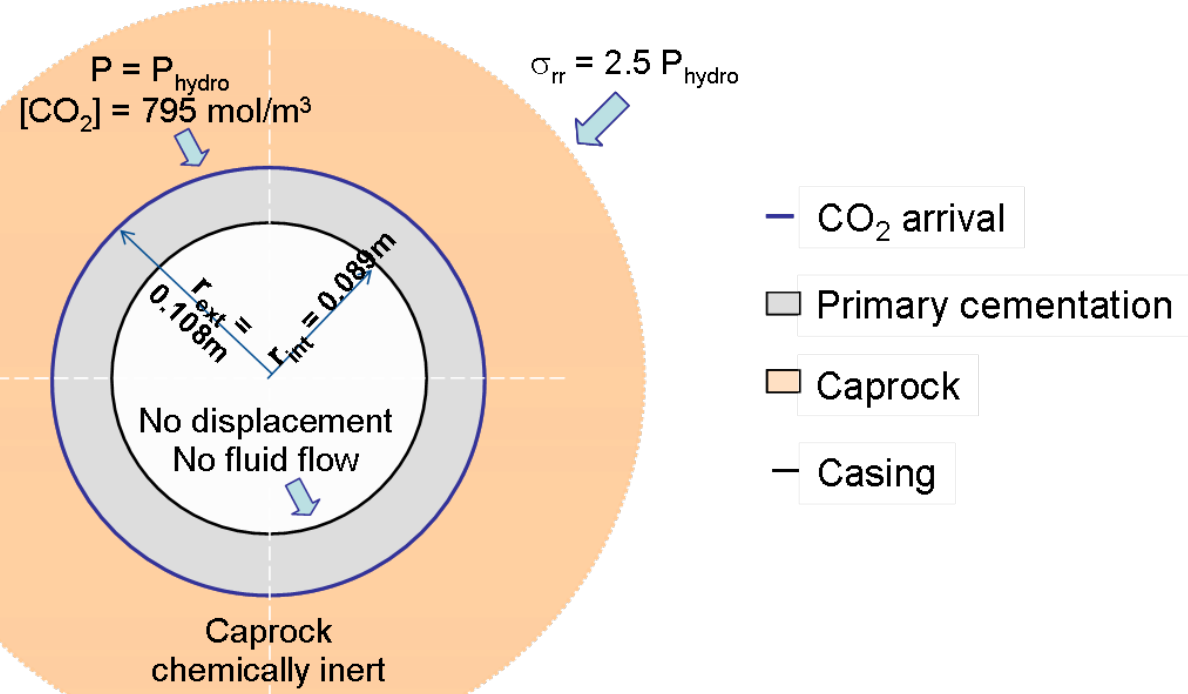

Figure 2: Boundary conditions 


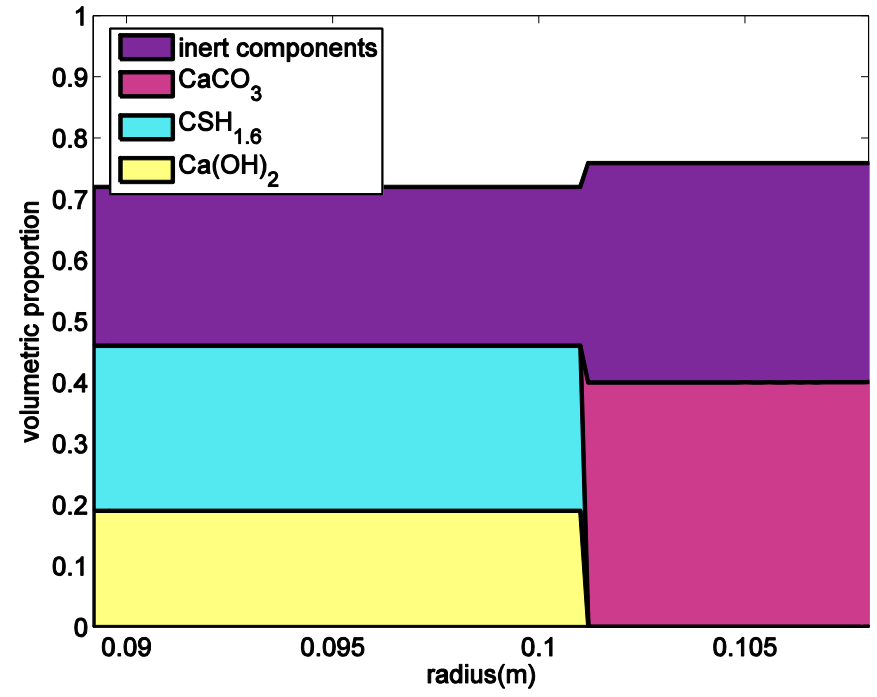

(3.a) at 10 days

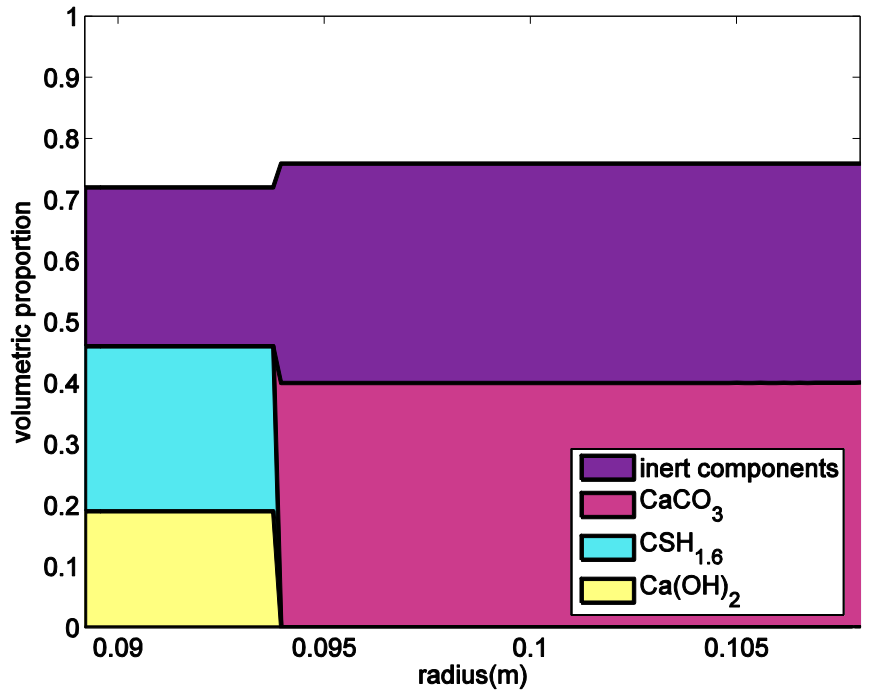

(3.b) at 40 days

Figure 3: Spatial repartition of molar quantities of the $\mathrm{CH}, \mathrm{C}-\mathrm{S}-\mathrm{H}$ and $\mathrm{CaCO}_{3}$ under $\mathrm{CO}_{2}$ injection at different times 


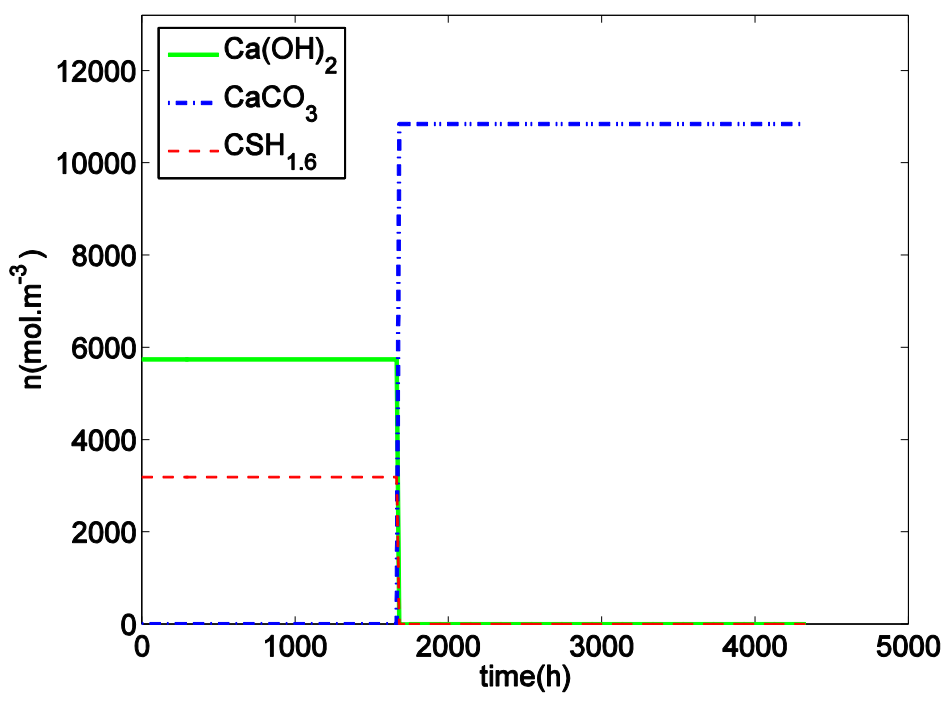

Figure 4: Evolutions of molar quantities of the $\mathrm{CH}, \mathrm{C}-\mathrm{S}-\mathrm{H}$ and $\mathrm{CaCO}_{3}$ under $\mathrm{CO}_{2}$ injection according to time in $r=$ rint

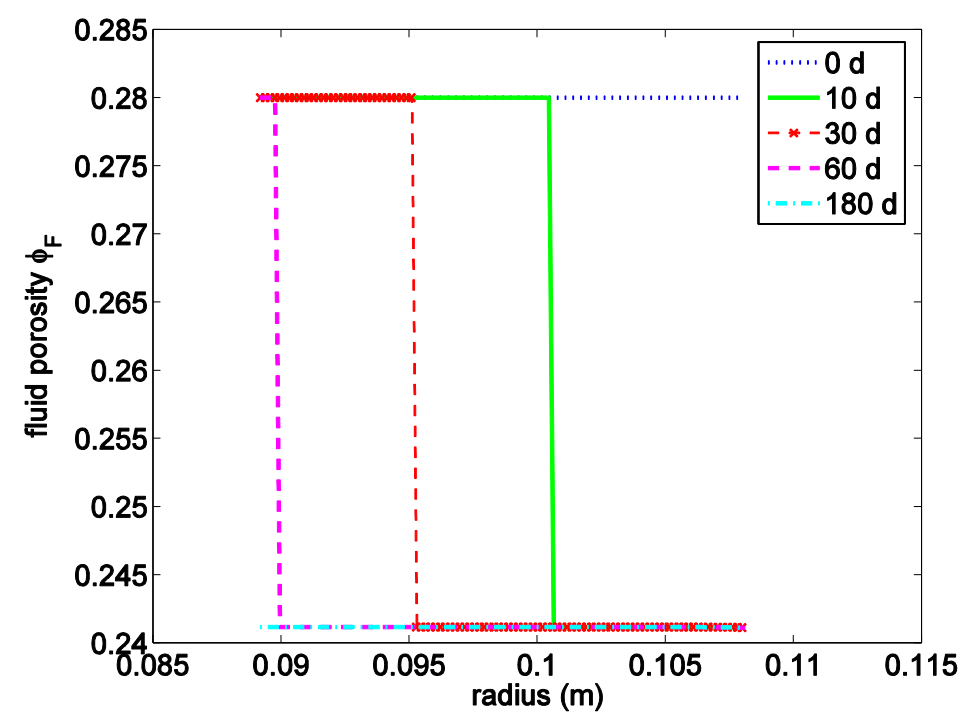

Figure 5: Spatial evolution of the porosity in the cement sample during 180 days of exposure to the $\mathrm{CO}_{2}$ at several times 


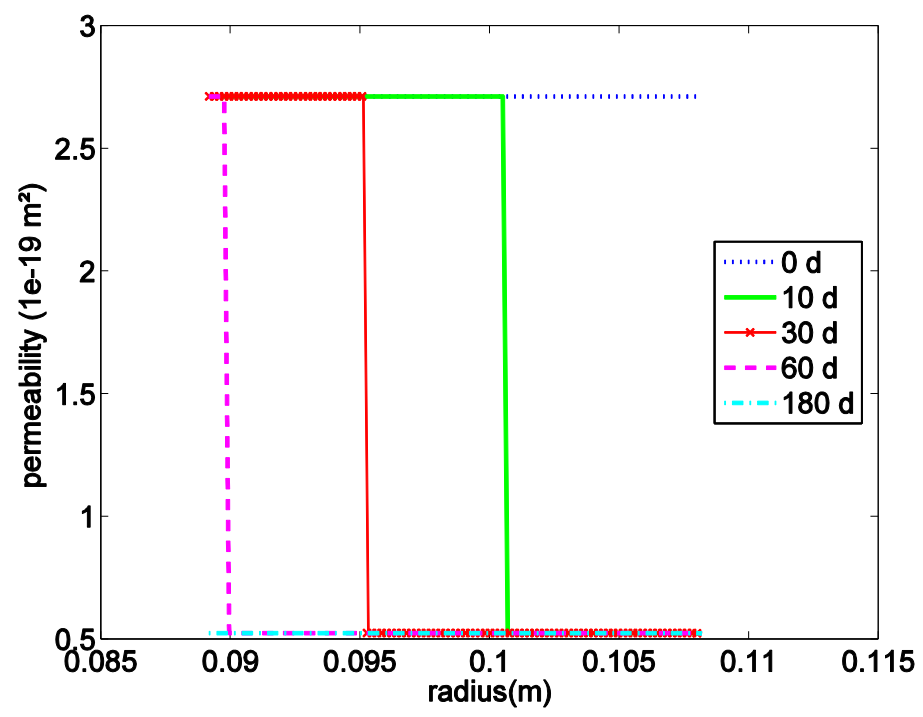

Figure 6: Spatial evolution of the permeability in the cement sample during 180 days of exposure to the $\mathrm{CO}_{2}$ at several times

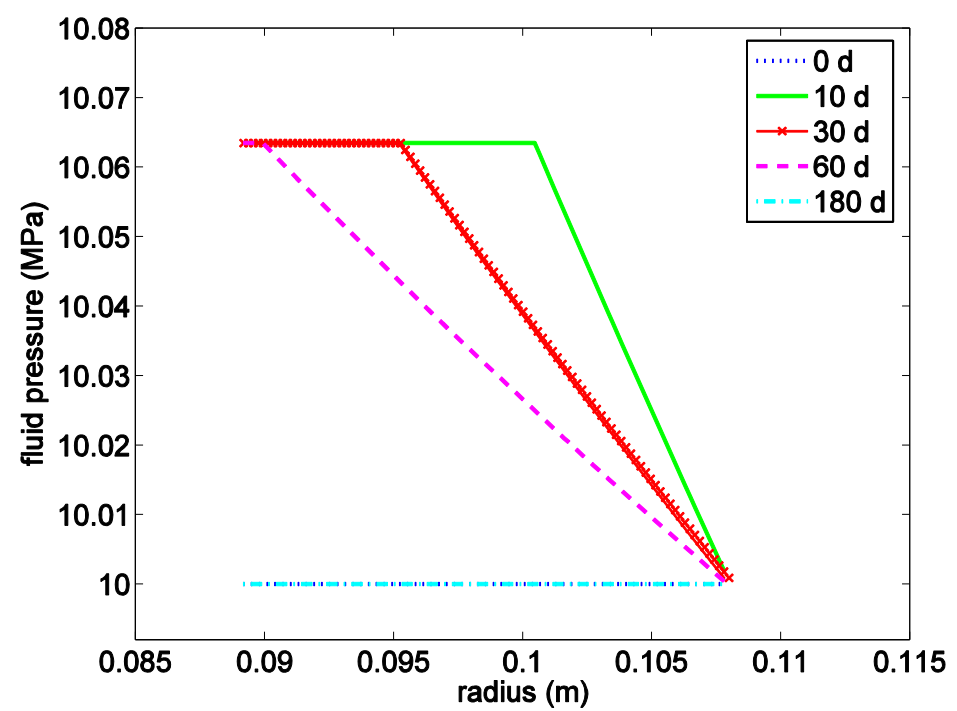

Figure 7: Spatial evolution of the fluid pressure in the cement sample during 180 days of exposure to the $\mathrm{CO}_{2}$ at several times 


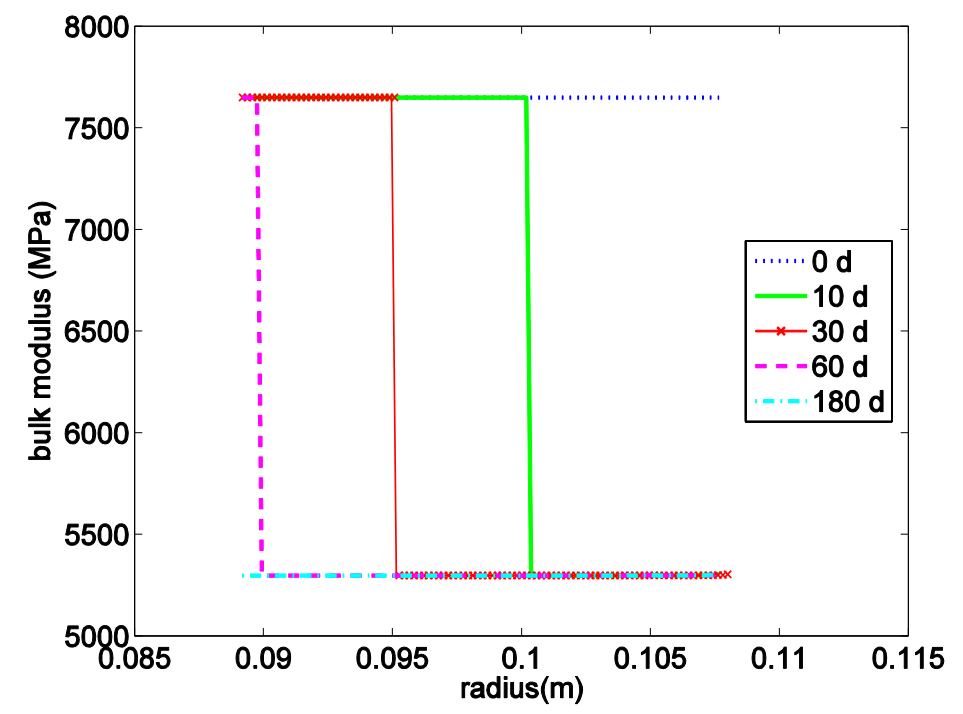

Figure 8: Spatial evolution of the bulk modulus in the cement sample during 180 days of exposure to the $\mathrm{CO}_{2}$ at several times

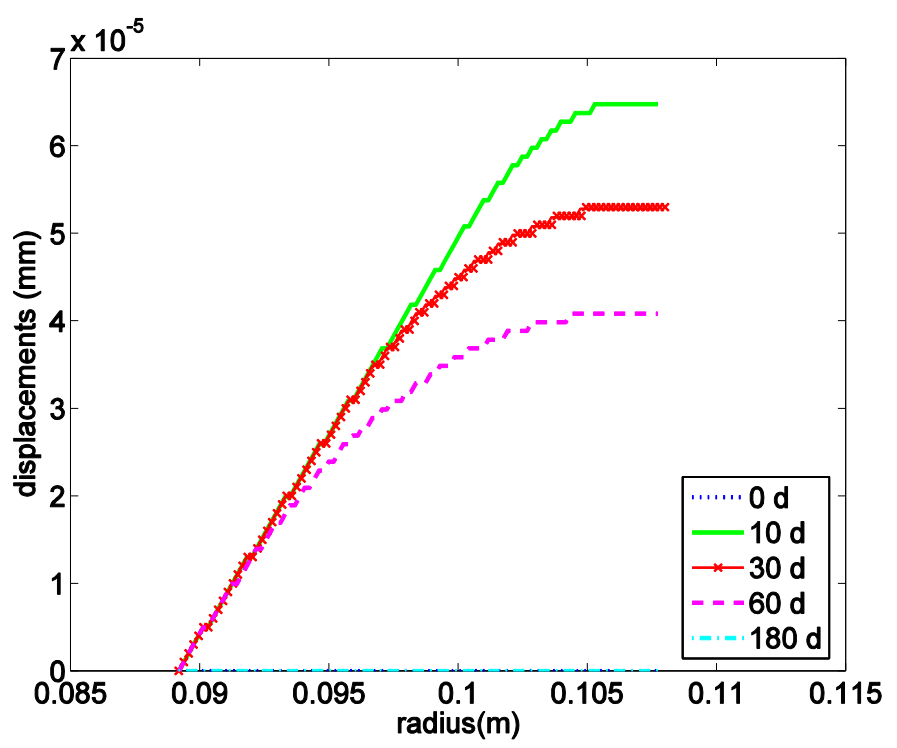

Figure 9: Spatial evolution of the displacements in the cement sample during 180 days of exposure to the $\mathrm{CO}_{2}$ at several times 


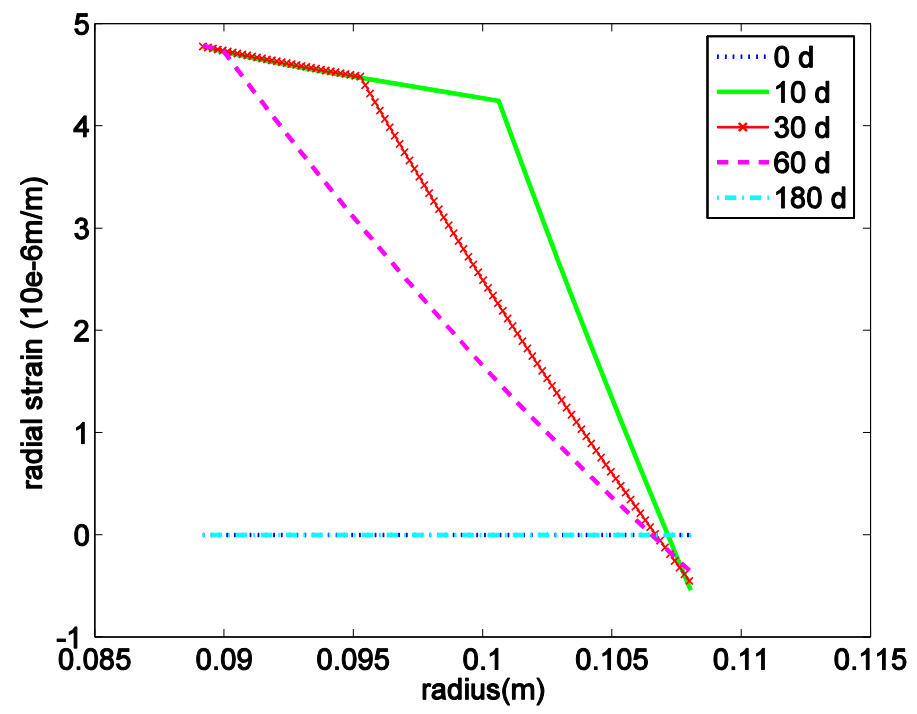

Figure 10: Spatial evolution of the radial strains in the cement sample during 180 days of exposure to the $\mathrm{CO}_{2}$ at several times

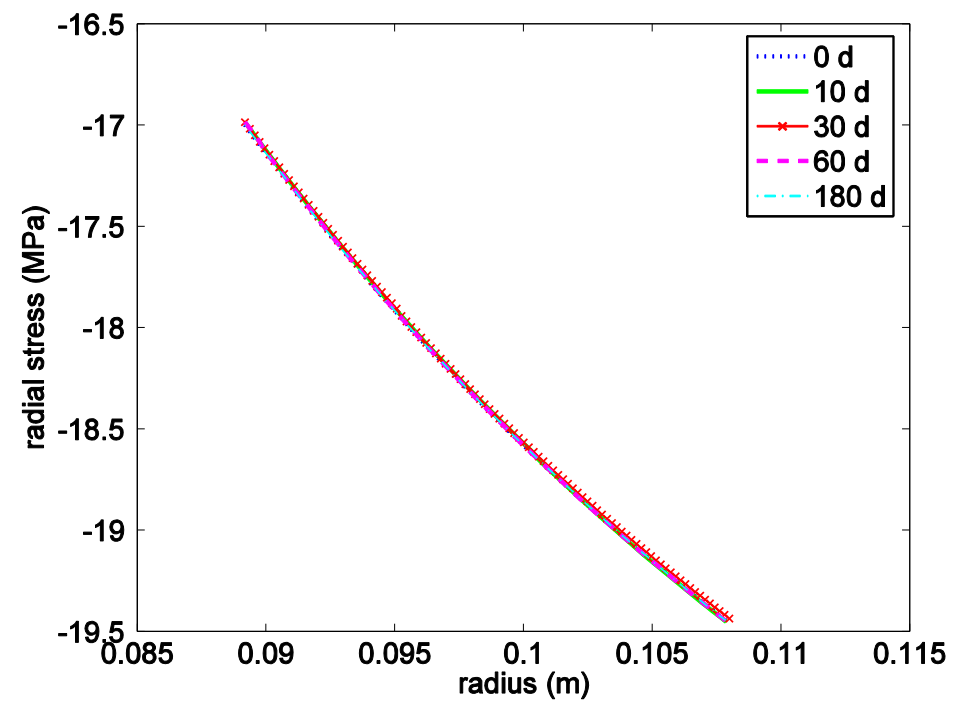

Figure 11: Spatial evolution of the radial stress in the cement sample during 180 days of exposure to the $\mathrm{CO}_{2}$ at several times 


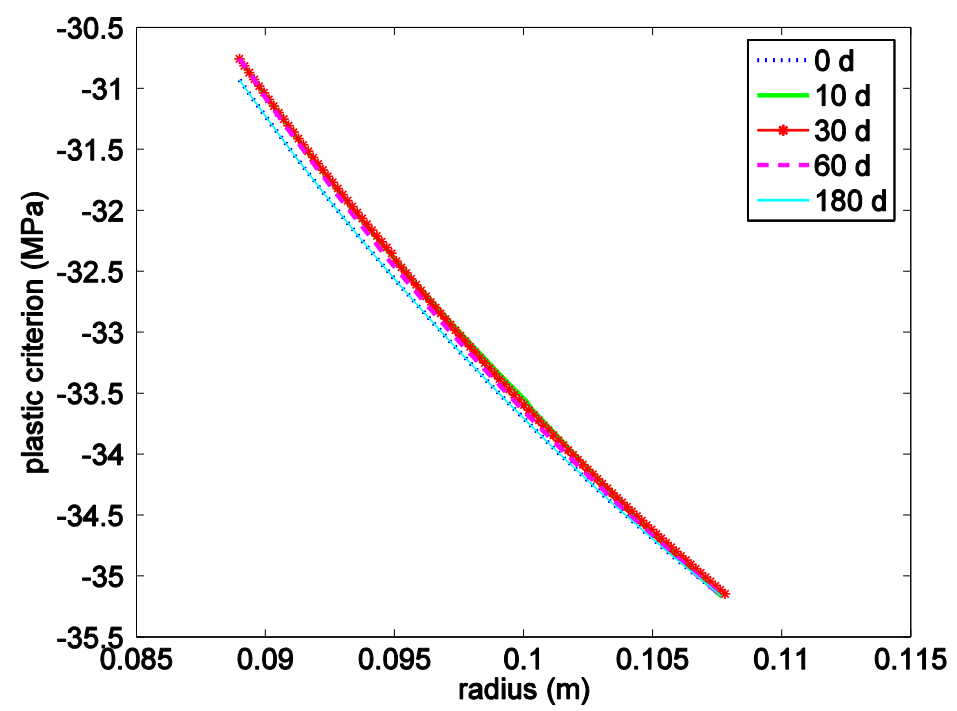

Figure 12: Spatial evolution of the yield criterion in the cement sample during 180 days of exposure to the $\mathrm{CO}_{2}$ at several times

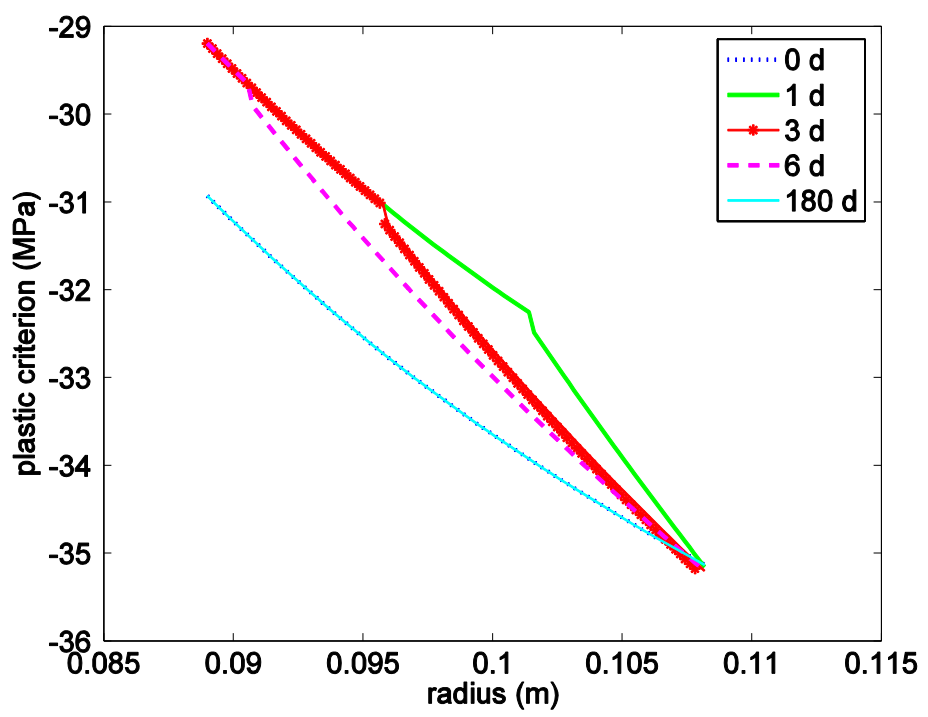

Figure 13: Spatial evolution of the yield criterion in a cement sample with $\mathrm{d}_{0}=4.6510^{-9} \mathrm{~m}^{2} \cdot \mathrm{s}^{-1}$ during 180 days of exposure to the $\mathrm{CO}_{2}$ at several times 


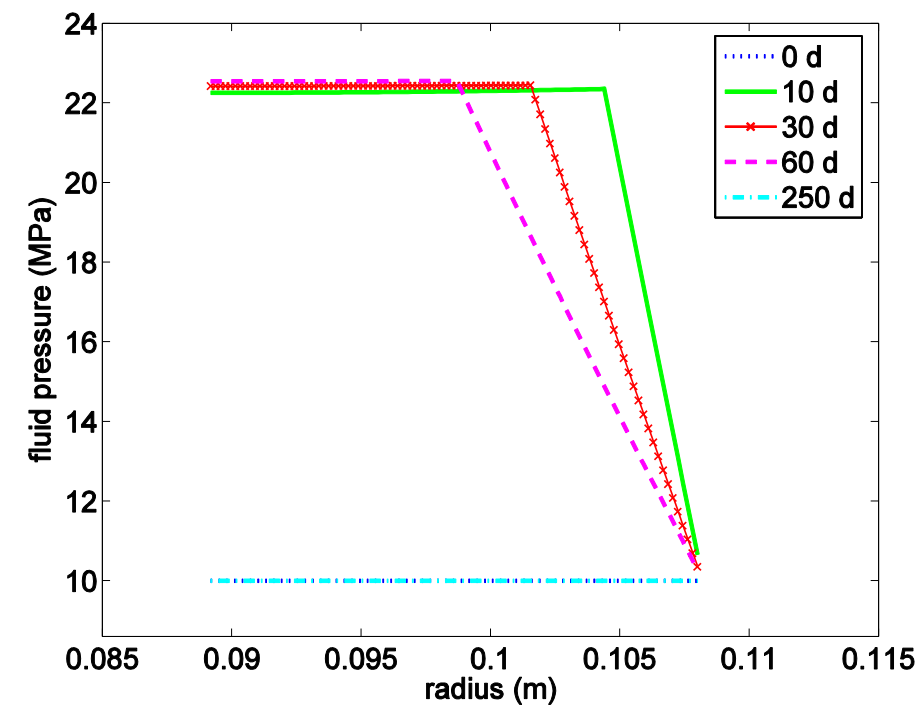

Figure 14: Spatial evolution of in-pore fluid pressure during 250 days of $\mathrm{CO}_{2}$ exposure with an initial porosity of $18 \%$

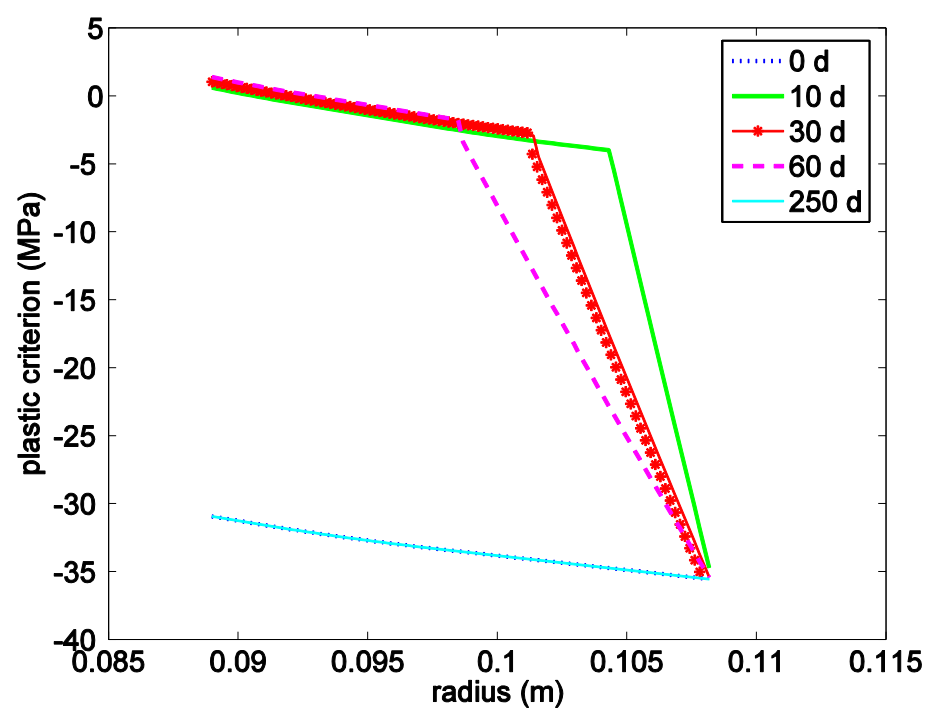

Figure 15: Spatial evolution of the yield criterion during 250 days of $\mathrm{CO}_{2}$ exposure with an initial porosity of $18 \%$ 